\title{
DARK MATTER IN SPIRAL GALAXIES
}

\author{
P.C. VAN DER KRUIT \\ Kapteyn Astronomical Institute \\ P.O. Box 800, 9700 AV Groningen, the Netherlands \\ (vdkruit@astro.rug.nl)
}

\section{Introduction}

In this review I will concentrate on three questions concerning the issue of dark matter in spiral galaxies. In the first place (and for the most part) I will discuss the analysis of rotation curves and the question whether the "maximum-disk hypothesis" is valid. Then I will discuss independent measurements of the disk mass distribution from the thickness of stellar and HI disks. Then follows a review of estimates of the flattening of the dark halos and a short discussion on the possible nature of the dark matter.

At the outset I state that the existence of dark halos is in my opinion beyond doubt. The observation of flat rotation curves using the $21-\mathrm{cm}$ line of HI up to radii well beyond (and often much beyond) the stellar disks establish the presence of gravitating matter out these large radii essentially as a certainty. The fact that these rotation curves are flat out to the last measured point furthermore indicates that the extent of these dark halos is in any case larger than that of the observable HI. For this review I will not discuss therefore total masses of spiral galaxies, but at most the total mass contained within some defined galactocentric distance. A recent review on the subject has also been given by Freeman (1993).

There have been proposals, that the flat rotation curves point not to unknown gravitating matter but instead to a breakdown of Newtonian gravitatonal theory, such as in Milgrom (1988). Such theories attribute the flat rotation curves to departures from the classical form of Newton's gravitation law such that the observed light distributions, when equated to a density distribution can explain the form of the rotation curve without invoking additional matter. I consider the main evidence against this the fact that the shape (but of course not amplitude) of rotation curves in the 
optically visible part show no correlation with morphological type over the whole range from bulge-dominated Sa's to disk-dominated Sc's (e.g. Rubin et al., 1985). As an extreme example I recall the case of the two galaxies NGC 891 and NGC 7814 (for a more detailed discussion see van der Kruit, 1983). In rough terms, NGC 891 has $80 \%$ of its light in an exponential disk with a radial scalelength of about $4.9 \mathrm{kpc}$ and $20 \%$ in a bulge with effective radius $2.3 \mathrm{kpc}$ (van der Kruit and Searle, 1981b). NGC 891 then has a half-light radius of about $5.5 \mathrm{kpc}$. NGC 7814 on the other hand is bulge-dominated, having about $90 \%$ of its light in a bulge with an effective radius of $2.2 \mathrm{kpc}$ and the rest in a disk with a radial scalelength of about 8 kpc (van der Kruit and Searle, 1982). Therefore in NGC 7814 the half-light radius is only about $2.3 \mathrm{kpc}$.

In spite of these very different central concentrations of light, both galaxies have essentially identical rotation curves. Due to the absence of HI these are not known within the central arcmin (about $3 \mathrm{kpc}$ in NGC 891 and about $4 \mathrm{kpc}$ in NGC 7814), but beyond that both rotation curves are essentially identical and flat at about $220 \mathrm{~km} / \mathrm{s}$ out to the edge of the optical disk at about $20 \mathrm{kpc}$. This means that the light distributions are no guide whatsoever to the shape of the rotation curves; non-Newtonian theories of gravity would predict grossly different rotation curves. In itself these facts also seem to suggest that even within the optical boundaries dark matter must make a significant contribution to the gravitational field in conventional theory.

\section{Rotation Curves and the Maximum Disk Hypothesis}

The critical part in analyzing rotation curves is the calculation of the contribution from the observed luminous components. For this these components are assumed to have the same $\mathrm{M} / \mathrm{L}$ at all positions. There are well defined (but sometimes tedious) equations to convert the observed surface brightness distributions in the disk and bulge into expected rotation curves (see for example van der Kruit, 1989, p. 212 for a summary). The contribution of the gas is derived from the observed HI distribution (if possible supplemented by an estimate of the molecular hydrogen from CO-maps) and corrected for the normal abundance of helium. The mass of the dust is usually neglected (probably correctly so). Then a spherical dark halo is added; this must have the property that at large radii it tends to a flat rotation curve of a density distribution proportional to $R^{-2}$. Earlier on the equations for the isothermal sphere have been used for this, but for numerical simplicity nowadays one usually uses a density distribution of the form $\rho(R)=\rho_{o} /\left[1+\left(R / R_{c}\right)^{2}\right]$. All these contributons have to be added in quadrature. 
This was applied by van Albada et al. (1985) to the spiral NGC 3198 where the measurable extends out to about $30 \mathrm{kpc}$ (or about 11 exponential scalelengths of the optical disk; or about $2.5 R_{25}$, the radius of the face-on corrected isophote at $25 \mathrm{~B}$-mag $\operatorname{arcsec}^{-2}$ ). They showed that there are enough parameters to arrive at reasonable fits for any M/L of the disk by adjusting the core-radius $R_{c}$ of the dark halo. Actually, they could reproduce the rotation curve with no disk at all! They then proposed the "maximum disk hypothesis", which selects the model with the highest $\mathrm{M} / \mathrm{L}$ of the disk possible; this also means that the disk gravitational field dominates all contributions to the observed rotation curve within the optical disk. A discussion of the validity of this assumption will be the main issue of this review, because of its importance in these analyses.

In a recent Groningen thesis, Broeils (1992) has collected rotation curves and optical photometry on a sample of 23 galaxies, all with extended HI. He made maximum disk fits to these rotation curves and derived various relations. First, he found that the integrated M/L (here and what follows these are in the B-band unless noted otherwise) to be generally in the range 10 to 20 out to the maximum measured radius in the rotation curve. This number (contrary to most others discussed in what follows) is not very sensitive to the assumption of maximum disk (only the flattening of the mass distribution enters into it).

A useful parameter is the ratio of dark to luminous mass out to a welldefined, uniform radius such as $R_{25}$ or $R=7 h$ (seven radial scalelengths of the optical disk). He found interesting properties, such as that the ratio of dark to luminous mass within such radii correlated well with the maximum speed in the rotation curve or integrated magnitude (these are the same thing in view of the Tully-Fisher relation) in the sense that it decreased with increasing maximum rotation velocity and integrated luminosity. Also it increased towards later types (galaxies classified later than Sc usually are dwarfs with low total luminosity). This is interesting, but the question remains to what extent this is an artifact of the maximum disk fits.

The question then is: Could the contribution of the disk (and the disk $\mathrm{M} / \mathrm{L}$ ) be overestimated in galaxies with large amplitude rotation curves? If this amplitude is large, the maximum disk hypothesis naturally gives rise to a large disk surface density and therefore a large disk M/L (after all these are all major spirals that conform to Freeman's law of a quite uniform central surface brightness of the disk). This in turn would give a small ratio of dark to luminous matter within the disk radius. Since late type spirals usually are small and blue and have small amplitude rotation curves, the maximum disk hypothesis would naturally give rise to relations where the disk $M / L$ is small for late-type and relatively blue galaxies, as indeed found by Broeils. Now, certainly the maximum disk hypothesis 
could be correct and apply to all galaxies, and Broeils indeed finds the correlations predicted here. He also correctly points out that simple (bimodel initial mass function) synthesis models are consistent with his result. But it is also well-known that these models are very uncertain in predicting the $M / L$, since $M$ depends on low-mass stars and $L$ on high-mass stars and the result depends really only on the assumed initial mass function and can easily be changed without violating any observations.

The analysis of Broeils gives a wide range of the ratio of dark-toluminous mass within the optical radius $\left(M_{\text {dark }} / M_{\text {lum }}\right)_{R_{25}}$ from 6 to 0.1, correlating well with a range of disk mass-to-light ratio $(M / L)_{d i s k}$ from 1 to 6 and an observed maximum rotation velocity $V_{\max }$ from 50 to 325 $\mathrm{km} / \mathrm{s}$ :

$$
\begin{gathered}
\left(\frac{M}{L}\right)_{\text {disk }}=(0.014 \pm 0.003) V_{\text {max }}+(0.72 \pm 0.60), \\
\left(\frac{M_{\text {dark }}}{M_{\text {lum }}}\right)_{R_{25}}=(2.37 \pm 0.39)-(0.42 \pm 0.12)\left(\frac{M}{L}\right)_{\text {disk }} .
\end{gathered}
$$

This does not necessarily mean that the maximum disk hypothesis does not hold, but only that naturally expected relations that could be artifacts, do indeed result. The ratio of dark to luminous mass within the optical radius is $\log \left(M_{\text {dark }} / M_{\text {lum }}\right)_{R_{25}}=-0.1 \pm 0.4$ in Broeils' analysis.

Alternatively, we can assume that disks have very similar $M / L$ values, for example 2 in Broeils' fits. Then $\log \left(M_{\text {dark }} / M_{l u m}\right)_{R_{25}}=-0.2 \pm 0.2$ with a much smaller variation, while the dependence on $V_{\max }$ essentially disappears. This is interesting, since it does not follow naturally from the assumption of a constant disk $\mathrm{M} / \mathrm{L}$ that this variation should be small and independent of the maximum rotation velocity.

So, what evidence do we have for the maximum disk hypothesis? This has been discussed also recently by Freeman (1993). In that review he showed results of the Buchhorn and Mathewson sample of about 550 spirals, for which they obtained optical (emission line) rotation curves and I-band photometry. It then turned out that for $97 \%$ of these galaxies these rotation curves (which of course do not go far out, generally not even out to $R_{25}$ ) could be reproduced simply by the rotation curve from the photometry and that with a fairly similar disk M/L for all systems. The latter is interesting, but can equally well be interpreted as saying us that, at least that sample of galaxies, the ratio of dark-to-luminous mass within the optical boundaries is a constant, lowering their derived disk M/L's with a uniform factor.

Most importantly, Freeman pointed to he fact that these predicted rotation curves from the luminosity profiles showed features ("wiggles") that are indeed observed in the slit spectra. Kent (1986) was the first to draw attention to such wiggles that were predicted from his photometry, although 


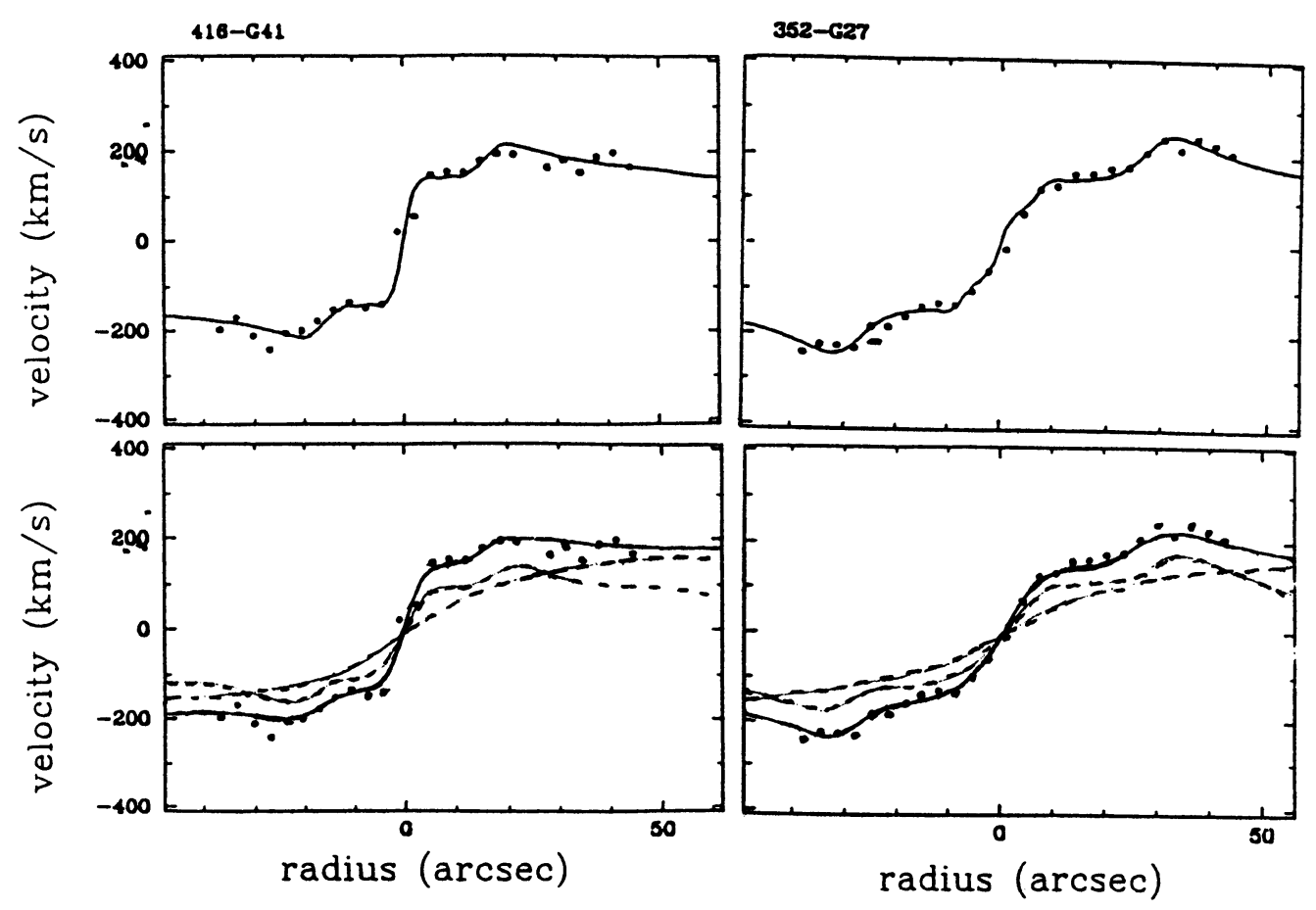

Figure 1. The rotation curves over the optical disks of two spirals from the Buchhorn and Mathewson sample. The panels on the left give the curves fitted by a rotation curve derived from the light distribution and a constant $M / L$ ratio, but no dark halo (maximum disk models). The right-hand panels show the predicted rotation curves when about half the mass within the optical disk is put in a dark halo (and the M/L ratio of the disk arbitrarily decreased by a factor 2 compared to the other panels). The amplitude of the features have of course decreased, but this excersize shows that fits at least equally good can be obtained with and without a substantial contribution from a dark halo within the optical radius (after Freeman, 1993).

Kalnajs (1983) had already shown that optical rotation curves (so extending not even out to $R_{25}$ ) could be reproduced from the photometry without a dark halo. Freeman showed two dramatic examples where all details in the rotation curves were reproduced by those in the luminosity distributions and concluded that the contribution that the dark halo makes to the inner rotation curve remains small as we go from the largest to the smallest spirals. How secure is this conclusion (clearly it is a valid, but not necessarily unique interpretation of the data)?

In order to check this I arbitrarily decreased the M/L of the disk in these two galaxies, as assumed in the Buchhorn and Mathewson fit, by a factor 2 (that means decreasing the rotation curves from the disks alone by 
a factor $\sqrt{2}$ ) and added a dark halo with the usual equation for the density and resulting rotation curve as given above. Another way of expressing this is to say that the mass is redistributed such that roughly only half is in the disk and half in a halo with a smooth density distribution. It admittedly does mean that the parameter $R_{c}$ has to be chosen such that the "haloalone" rotation curve has a general shape not too different from that of the "disk-alone". This of course then is far from a maximum disk fit. Certainly, the wiggles are less prominent (Fig. 1), but in these "eye-ball" judgements the fit certainly looks equally good (if not better, as I would be tempted to say). The conclusion is then that the presence of these wiggles and their correspondence with features in the luminosity profiles is far from strong evidince for the maximum disk hypothesis.

\section{Thickness of Stellar Disks and HI Layers}

In this section I will discuss independent measurements of the M/L of disks from thicknesses of stellar and HI layers and further evidence concerning the validity of the maximum disk hypothesis for all galaxies. First I will discuss measurements of the $\mathrm{z}$-dynamcis of stellar disks.

Since the early measurements of the radial distribution of the stellar velocity dispersion in galactic disks by van der Kruit and Freeman (1986), Bottema (1993 and references therein) has measured and collected the data in 12 spirals. In general these velocity dispersions fall off with radius with an exponential scalelength twice that of the surface brightness. At least in the vertical direction this is consistent with the observed constant scaleheight in galactic disks with radius (van der Kruit and Searle, 1981a) if the M/L has no radial dependence. Bottema has derived a relation between a fiducial velocity dispersion and the integrated magnitude of the old disk population (that is after subtraction of a young population I on the basis of the colors) and the maximum in the rotation curve. In order to compare highly inclined galaxies (where on measures velocity dispersions in the plane) and face-on ones (where the $\mathrm{z}$-velocity dispersion is measured along the line of sight) he plots for the latter the velocity dispersion at one scalelength and for the former at the center. If the axis ratio of the velocity ellipsoid is everywhere similar as in the solar neighborhood, then with the exponential fall-off mentioned these should be comparable. Indeed he finds for both samples the same relations, confirming the constancy of this axis ratio within and between galactic disks.

For his sample this fiducial velocity dispersion increases from about 30 $\mathrm{km} / \mathrm{s}$ at $M_{B, d i s k}=-18.5$ and $V_{\max }=120 \mathrm{~km} / \mathrm{s}$ to about $90 \mathrm{~km} / \mathrm{s}$ at $M_{B, \text { disk }}=-22$ and $V_{\max }=300 \mathrm{~km} / \mathrm{s}$. This can be understood (see van der Kruit, 1989, p. 198) as follows: Toomre's stability parameter $Q \propto\left\langle V_{R}^{2}\right\rangle \kappa \sigma^{-1}$, 
where $\sigma$ is the surface density. The epicyclic frequency for a flat ration curve is $\kappa \propto V_{\text {max }} R^{-1}$. Using an exponential disk, $\sigma \propto \mu_{o}(M / L) \exp (-R / h)$ with $\mu_{o}$ the central face-on surface brightness and the Tully-Fisher relation $L \propto V_{\max }^{4}$, these equations combine to for the radial velocity dispersion at one scalelength as

$$
\left\langle V_{R}^{2}\right\rangle_{h}^{1 / 2} \propto \mu_{o}(M / L) Q V_{\max } \propto \mu_{o}(M / L) Q L^{1 / 4} .
$$

This nicely fits his relations. Now for these spiral we have $\mu_{o}$ about constant, so that this shows that the product of (M/L) and $Q$ needs to be constant. As can be seen from Bottema's analysis the product of these two parameters is about 5 . From n-body simulations of secular heating in disks, many studies (e.g. Athanassoula and Sellwood, 1986) predict an equilibrium value for $Q$ of about 2 to 2.5 . This would imply values for the $\mathrm{M} / \mathrm{L}$ of the old disk of also about 2 to 2.5 . We then have here evidence that unless $Q$ would vary greatly between disks (which is not theoretically appealing) the M/L of disks is likely to be constant as well between disks, which contradicts the outcome of maximum disk fits.

Bottema went one step further as follows. Using the equation for the exponential, constant thickness disk with $\rho(R, z)=\rho(0,0) \exp (-R / h)$ $\operatorname{sech}^{2}\left(z / z_{o}\right)$, that for the velocity dispersion in the z-direction $\left\langle V_{z}^{2}\right\rangle_{R=0}^{1 / 2}=$ $\sqrt{\pi G \sigma_{o} z_{o}}$, and that for the maximum rotation velocity in a self-gravitating exponential disk $V_{\text {max }, \text { exp }}=0.88 \sqrt{\pi G \sigma_{o} h}$, one gets

$$
V_{\text {max }, \text { exp }}=0.88\left\langle V_{z}^{2}\right\rangle_{R=0}^{1 / 2} \sqrt{h / z_{o}} .
$$

The precise numerical constant has only a minor dependence on whether the $\operatorname{sech}^{2}$ vertical density distribution is chosen or some other one (see discussion by Bottema). He then finds that his observed relations can only be fitted without invoking a dark halo, if $h / z_{o}$ is of order 15 , while the observed value in edge-on spirals is more like 5 or so. This discrepancy shows that the amplitude of the rotation curve within the optical boundaries cannot be provided by the disk alone. The disk rotation curve has an amplitude of on average only $2 / 3$ of that observed and Bottema therefore concludes that spiral galaxies are in general not maximum disk.

The evidence from HI layers has been reviewed elsewhere in detail (van der Kruit, 1989, p. 218 and further). For NGC 891 the disk-alone rotation curve has a maximum of $100 \pm 20 \mathrm{~km} / \mathrm{s}$ and it should be stressed that this involves no assumed M/L ratio. At this radius of about 2 scalelengths, where the rotation curve of an exponential disk reaches its maximum, the bulge-alone rotation velocity would be about $100 \mathrm{~km} / \mathrm{s}$, so the expected rotation velocity (allowing also for the gas) is about $150 \pm 25 \mathrm{~km} / \mathrm{s}$. The observed rotation velocity in NGC 891 is $225 \pm 10 \mathrm{~km} / \mathrm{s}$, so we have here 
direct evidence that this galaxy at least cannot be maximum disk. Kuijken (this Symposium) has given a comparable discussion for our Galaxy and comes to the same conclusion there.

\section{Shapes of Dark Matter Halos}

The studies of the thickness of HI layers and so, as in the preceding section, only show that the dark matter cannot reside in the disks, but do not put any useful limits on the flattening of these dark matter halos.

The first is from kinematics of polar ring galaxies. There the rotation velocity in the ring is quite similar to that measured in the main body of the galaxy itself. This means that the potential field is very close to spherical, but it is much more difficult to obtain from that useful limits on the flattening of the halo. After all, even a pure disk has equal-potential surfaces that at larger $\mathrm{z}$-distances quickly become spherical. Whitmore et al. (1987) did a very thorough study in three polar ring galaxies and found that the potential field was flattened only slighly with axis ratio of 0.8 to 1.0. However, it proved to be difficult to determine from this reliable limits on the flattening of the distribution of the gravitating matter, since a wide range of flattenings could accomplish the derived flattenings of the potential field.

This situation has become somewhat puzzling (and interesting) by the recent study of Sackett et al. (1994b). In the polar ring galaxy NGC 4650A they derive a density flattening with axis ratio 0.4 to 0.3 . The surprising thing is that this is very flat and very close to that of the central luminous body.

The second method uses the observed warps in the HI layers beyond the optical disks in spiral galaxies. These models usually involves the modeling of a warp of a disk that is initially tilted with respect to a dark matter halo. The timescale of the settling of this disk and its warp is the crucial point. Hofner and Sparke (1994) recently did such a modeling of observed warps in a few highly inclined spirals. In particular, they found a halo flattening of about similar to an E4 galaxy for NGC 2903 and one close to spherical for NGC 3198.

In a recent paper Sackett et al. (1994a) have found evidence for a faint, red halo in the light distribution of the edge-on spiral NGC 5907. Its distribution is such that it follows that of a dark halo as inferred from the rotation curve. This is a very tantalizing observation, although it needs confirmation in this galaxy and preferably also in others. The inferred color and M/L (about 450 in the R-band) are similar to those of low-metallicity late M-dwarfs and in agreement with older upper limits (see van der Kruit, 1987). However, it would be ironic that dark halos are made up entirely of 
sub-dwarfs just above the lower limit of main sequence hydrogen burning.

\section{References}

Albada, T.S. van, Bahcall, J.N., Begeman, K. and Sancisi, R., 1985, Ap. J. 295, 305

Bottema, R., 1993, A. $\mathscr{G}$ A. 275, 16

Broeils, A.H., 1992, Dark and visible matter in spiral galaxies, Ph.D. thesis, Groningen University

Freeman, K.C., 1993, Physics of Nearby Galaxies: Nature or Nurture?, ed. R.X. Thuan, C. Balkowski and J. Tran Thanh Van, Editions Frontières, p. 201

Hofner, P. and Sparke, L.S., 1994, Ap. J. , in press

Kalnajs, A., 1983, Internal Kinematics and Dynamics of Galaxies, IAU Symposium 100, ed. E. Athanassoula, Reidel, p. 87

Kent, S., 1986, A. J. ]bf 91, 1301

Kruit, P.C. van der and Freeman, K.C., 1986, Ap. J. 303, 556

Kruit, P.C. van der, 1987, Dark Matter in Galaxies, IAU Symposium 117), ed. J. Kormendy and G.R. Knapp), p. 415

Kruit, P.C. van der and Searle, L., 1981a, A. \& A. 95, 105.

Kruit, P.C. van der and Searle, L., 1981b, A. \& A. 95, 116

Kruit, P.C. van der and Searle, L., 1982, A. \& A. 110, 79

Kruit, P.C. van der, 1983, Proc. Astron. Soc. Australia 5, 136

Kruit, P.C. van der, 1989, The Milky Way as a Galaxy, G. Gilmore, I.R. King and P.C. van der Kruit, University Science Books

Milgrom, M., 1988, Ap. J. 333, 68

Rubin, V.C., Burstein, D., Ford, W.K. and Thonnard, N, 1985, Ap. J. 289, 81

Sackett, P.D., Morrison, H.L., Harding, P. and Boroson, T.A., 1994a, Nature, 370, 441

Sackett, P.D., Rix, H.-W., Jarvis, B.J. and Freeman, K.C., 1994b, Ap. J. , in press

Whitmore, B.C., McElroy, D. and Schweizer, F., 1986, Ap. J. 314, 439

CASERTANO: Before we discount the relations found by Broeils and their implications on the maximum disk hypothesis, I would like to point out that similar correlations were found previously by Jacqueline van Gorkom and myself, with the important difference that we avoided any model-dependent rotation curve decomposition by using the properties of the rotation curve itself. While this does not address directly the maximum disk hypothesis, the fact that similar correlations are found with or without rotation curve decomposition would seem to support the validity of the correlations themselves.

VAN DER KRUIT: These correlations involve those between total masses of dark haloes out to distances well beyond the optical radius. The total amount of dark matter so far out is only weakly dependent on the rotation curve decomposition and I do not take issue with relations involving total masses. What I was referring to were relations involving the amount of luminous matter as given by Broeils and those critically depend on decompositions and the assumptions of a maximum disk. So I agree that there are relations between dark matter mass out to the last measured point and other properties, but as soon as the mass of luminous matter is involved or the dark mass within the optical radius, we should be very careful. GERHARD: In the beginning of your talk you showed the rotation curve of NGC 7814 and that it cannot be explained by MOND non-Newtonian gravity, because the light distribution of the galaxy is so centrally concentrated. But the rotation curve also did not fit the (standard) Newtonian prediction from the light of the bulge system. What could be the explanation for this? 
VAN DER KRUIT: The example I showed assumed arbitrary M/L ratios dor disk and buge designed to provide the rotation velocity at larger radii. All I illustrated was the expected form, not the amplitude, of the rotation curve if no dark matter is assumed. Furthermore, there is no HI in NGC 7814 within 1 arcmin and we do not know the circular velocity there. My figure illustrated that we cannot expect similar rotation curves without dark matter (or in MOND) for the two different light distributions of NGC 891 and NGC 7814.

OLIVIERA: How many of the galaxies in your sample showed asymmetric rotation curves?

VAN DER KRUIT: In general asymmetries are minor, but present. The Broeils sample was selected for symmetric, well-behaved rotation curves in spirals with extended HI. M101, which does have large asymmetries in the HI distribution and velocity field, was not in the sample.

TAYLER: From what has been said there seems to be considerable uncertainty about how much dark matter there is in spiral galaxies. Do there remain galaxies for which the rotation curve is measured flat so far out that there is completely unambiguous evidence of dark matter?

VAN DER KRUIT: The evidence for dark matter is without doubt, as indicated by flat rotation curves in $\mathrm{HI}$ to often far beyond the optical disk. What I addressed was the matter of maximum disk models and question of how important a role the dark matter plays within the radius of the optical disk.

WHITE: Following up on a remark by Ken Freeman. If indeed the rotation properties of the visible parts of most disk galaxies are dominated by the observed stars, there should be a strong correlation of $\mathrm{M} / \mathrm{L}$ with other observed properties of the stellar population (e.g. colour). Is this the case?

VAN DER KRUIT: There is indeed some correlation in maximum disk fits between $\mathrm{M} / \mathrm{L}$ and color, and it can be fitted by models. But these are too arbitrary to use as supporting evidence due to the well-known effect that M/L depends on the number of low-mass stars providing the mass and the colors only on the massive stars.

MARGARET BURBIDGE: I was interested to see the recent work by Sackett et $a l$., in which they detected an extended red halo of stars around the edge-on spiral NGC 5907. I wonder whether you would comment further on this work, and their determination that the red luminosity falls of as $R^{-2}$, less steeply than any known luminous component of spiral galaxies.

VAN DER KRUIT: Their result is very interesting and this CCD work is consistent in general with older upper limits from photographic photometry. I cannot argue with the data, but clearly it needs confirmation. It puzzles me that the implication is that the dark matter would consist of stars with masses just (and only just) above the lower limit of hydrogen burning in sub-dwarfs. Also their data are consistent with $R^{-2}$, but in view of the large errors at these very faint levels presumably also with other radial dependences. I consider this observation intriguing, but in need of confirmation and extension to other galaxies. 